\title{
Growth and specification of the eye are controlled independently by Eyegone and Eyeless in Drosophila melanogaster
}

\author{
Maria Dominguez ${ }^{1}$, Dolors Ferres-Marco ${ }^{1}$, Francisco J Gutierrez-Aviño ${ }^{1}$, Stephan A Speicher ${ }^{1}$ \& \\ Monica Beneyto ${ }^{1,2}$
}

Control of growth determines the size and shape of organs. Localized signals known as 'organizers' and members of the Pax family of proto-oncogenes are both elements in this control. Pax proteins have a conserved DNA-binding paired domain, which is presumed to be essential for their oncogenic activity. We present evidence that the organizing signal Notch does not promote growth in eyes of D. melanogaster through either Eyeless (Ey) or Twin of eyeless (Toy), the two Pax6 transcription factors. Instead, it acts through Eyegone (Eyg), which has a truncated paired domain, consisting of only the C-terminal subregion. In humans and mice, the sole PAX6 gene produces the isoform PAX6(5a) by alternative splicing; like Eyegone, this isoform binds DNA though the $\mathrm{C}$ terminus of the paired domain. Overexpression of human PAX6(5a) induces strong overgrowth in vivo, whereas the canonical PAX6 variant hardly effects growth. These results show that growth and eye specification are subject to independent control and explain hyperplasia in a new way.

The distinctive shape of an organ or a limb is under genetic control, but what molecular mechanisms enable large fields of cells to form a particular shape and size? Control of growth is often linked to the establishment of localized signaling centers known as organizers in both invertebrates and vertebrates ${ }^{1-4}$. These organizers act as signaling centers that instruct, at long range, the growth and patterning of nearby cells. In widely different types of organs, the same set of signals (Notch, Hedgehog, Wnts, Dpp/BMP and FGFs) is used repeatedly to organize growth. This raises the question of how specificity is achieved.

Specificity could be achieved if the organizing signals activate different nuclear or transcription factors to mediate the specific growth response. A crucial prediction of this hypothesis is that the organspecific factor should be sufficient to mediate growth, even in the absence of activity of the upstream signal. Also, its aberrant expression, like the activity of the organizer itself, should cause deregulated growth.

Many tissues and organs acquire their identity through the differential expression of transcription factors of the Pax family, which is conserved from flies to humans ${ }^{5,6}$. Mutations in genes encoding Pax proteins not only disrupt organogenesis in fruit flies and mice ${ }^{6}$ but also cause congenital disease syndromes in humans ${ }^{5-10}$, characterized by loss or hypoplasia of particular organs. Moreover, a growing body of evidence implicates deregulated expression of $P A X$ genes in tissue-specific tumors in humans $5,6,11-15$. The association between developmental defects and oncogenesis suggests that members of this family may mediate organ-specific growth in response to generic organizing signals.
Pax proteins are defined by a conserved DNA-binding domain called the paired domain. Most have an octapeptide motif of unknown function, and several (e.g., Pax6; Fig. 1a) have a homeodomain ${ }^{6}$. The paired domain is thought to be necessary for oncogenesis ${ }^{16}$, but confirmation of this function in vivo remains elusive ${ }^{5,10,17}$, owing perhaps to difficulties in distinguishing tissue specification and growth defects and the use of alternative splicing in the mammalian $P A X$ genes.

The paired domain is a bipartite DNA-binding domain of 128 amino acids, consisting of $\mathrm{N}$-terminal and $\mathrm{C}$-terminal subdomains, each recognizing distinct DNA sites ${ }^{18-20}$. Although the $\mathrm{N}$-terminal and C-terminal subregions of the paired domain can bind DNA with high affinity when expressed separately, in the context of an intact paired domain, Pax proteins bind DNA preferentially through the $\mathrm{N}$-terminal subregion. The DNA-binding potential of the $\mathrm{C}$ terminus is only manifest when the N-terminal activity is blocked ${ }^{18-20}$. For instance, an alternative splicing event in the sole PAX6 gene in humans and rodents creates the isoform PAX6(5a) ${ }^{10,19}$, which lacks the normal paired domain activity owing to the inclusion of an extra exon in the N-terminal subregion (Fig. 1a). This isoform recognizes DNA exclusively through the C-terminal subregion of the paired domain. Likewise, alternative use of exons in the paired domains of $P A X 3$ and $P A X 8$ also results in the production of isoforms that use the C-terminal subdomain for DNA binding ${ }^{6,18-20}$. The purpose of masking the C-terminal activity in the intact paired-domain proteins is unclear because the biological functions of these isoforms are unknown.

${ }^{1}$ Instituto de Neurociencias, Consejo Superior de Investigaciones Científicas (CSIC) and Universidad Miguel Hernández, Campus de San Juan, Apartado 18, Sant Joan d'Alacant E-03550, Spain. ${ }^{2}$ Present address: Mental Health Research Institute, Department of Psychiatry, University of Michigan, 205 Zina Pitcher Place Ann Arbor, Michigan 48109, USA. Correspondence should be addressed to M.D. (m.dominguez@umh.es). 
The developing eye of the fruit fly $D$. melanogaster is ideal for studying the control of growth in vivo. First, the growth of the eye primordium (called the eye disc) depends on an evolutionarily conserved organizer (Fig. 1b) ${ }^{3,21-25}$. Second, the D. melanogaster genome has both classes of Pax transcription factors, but unlike mammalian genomes, these proteins are encoded by separate genes, enabling us to inactivate or activate each class individually.

The gene eyeless $(e y)^{26}$ and its paralog twin of eyeless $(t o y)^{27}$ encode canonical Pax6 products (Ey and Toy, respectively) and are the key players in the specification of eye identity. Ey and Toy trigger ectopic eyes when misexpressed ${ }^{27,28}$. Mouse Pax6 can substitute for $D$. melanogaster Ey in the formation of ectopic eyes ${ }^{28}$, and $D$. melanogaster Ey or Toy can induce vertebrate-type eyes in the frog embryo $^{29}$. The gene eyegone (eyg) encodes a Pax6-like protein (Eyg) $)^{10}$ with a truncated paired domain, lacking most of the $\mathrm{N}$ terminus ${ }^{30,31}$
(Fig. 1a). Biochemical studies showed that Eyg binds through the $\mathrm{C}$ terminus of the paired domain to a $5 \mathrm{aCON}$-like site ${ }^{30}$, which corresponds to the high-affinity binding site for mammalian Pax splice isoforms, such as PAX6(5a) ${ }^{18-20}$. Eyg is characterized as an eye-specifying factor $^{31-33}$, and overexpression studies suggested that Eyg and Ey might substitute for each other functionally ${ }^{31}$. Because the two proteins have distinct paired domain structures (Fig. 1a), however, they may have distinct roles during normal eye development.

Here we report that the Notch organizing signal promotes growth through the activation of eyg in the D. melanogaster eye. Overexpression of Eyg, but not Ey or Toy, consistently rescues the eye-growth shortfall of Notch mutants. Moreover, eyg is essential for eye growth but seems dispensable for specification of the eye. Finally, we report that the human PAX6(5a) splice isoform, which also lacks the N-terminal subdomain, induces strong overgrowth when

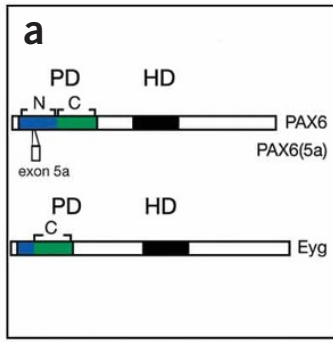

b
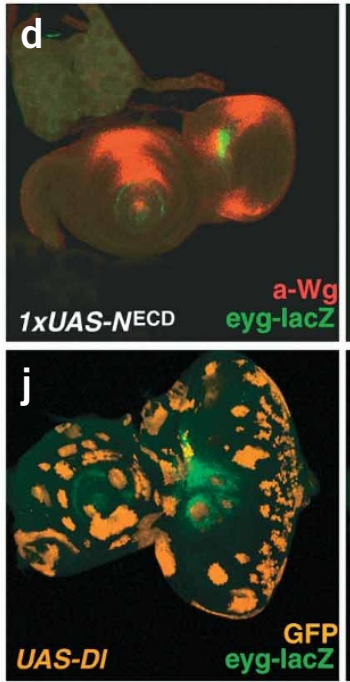

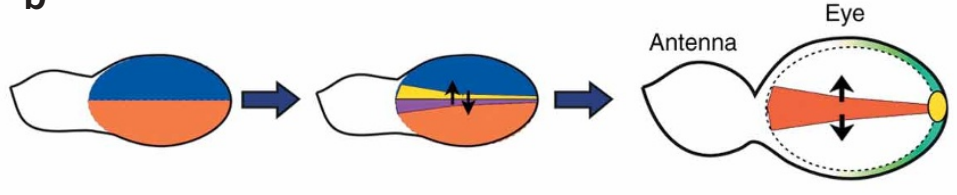

Iroquois -1 fringe
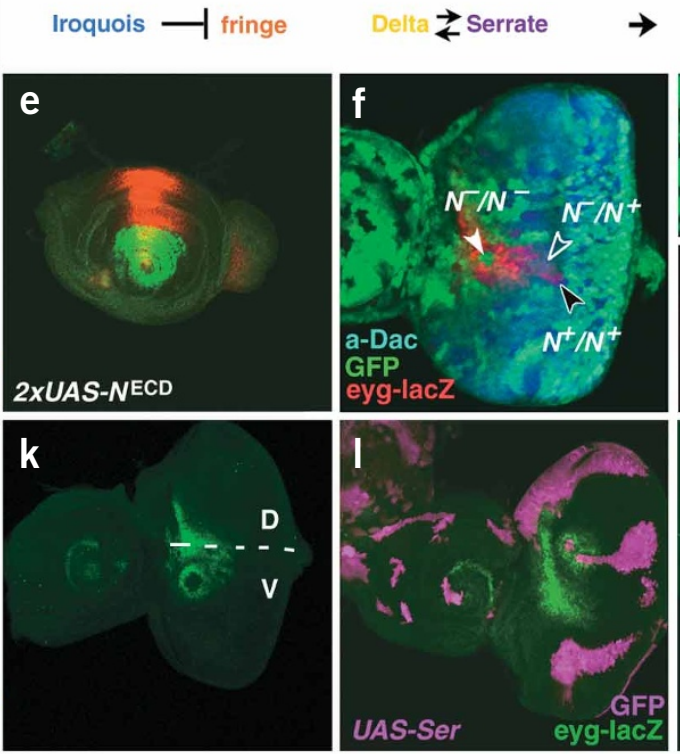

Notch activation
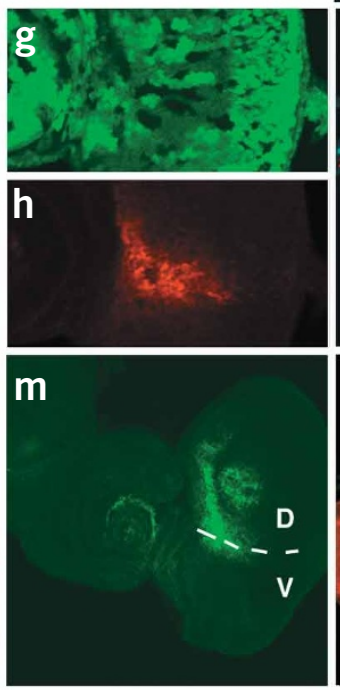
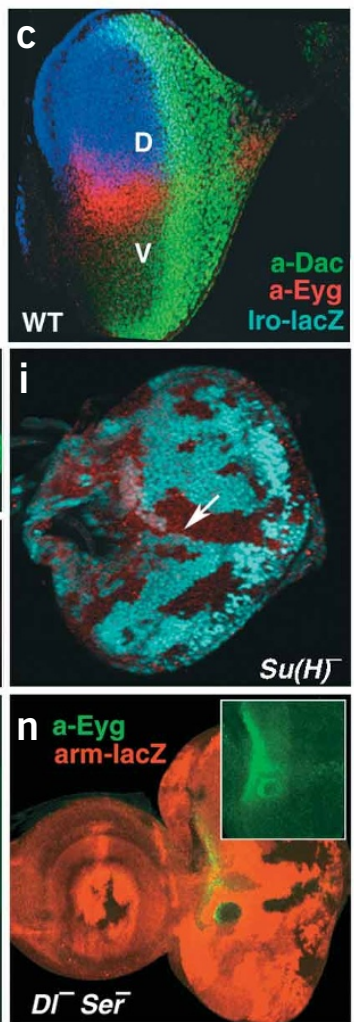

Figure 1 Notch signaling is necessary and sufficient to activate eyg expression in the D. melanogaster eye dorsal-ventral organizer. (a) Paired domain (PD) structure of the human PAX6 (top) and PAX6(5a) (middle) splice isoforms and fly Eyg protein (bottom). Eyg has a truncated paired domain and a Pax6-like homeodomain $(H D)^{10,30,31}$. (b) Schematics of the events that establish the dorsal-ventral organizer. The homeodomain Iroquois proteins (blue) repress the expression of the gene fringe (orange). fng cells are sensitive to Delta (yellow), a Notch ligand, and refractory to Serrate (purple), another Notch ligand, so that Serrate signals dorsal cells to express the gene Delta. Delta signals back to upregulate expression of Serrate in ventral cells. This results in the activation of Notch receptors at both sides of the fringe boundary (red) and the establishment of the growth-promoting organizer ${ }^{21-25}$. (c) Expression of the Eyg (red) transcription factor at mid-third larval stage is along the dorsal (D)-ventral (V) organizer as defined by the border of Iro-lacZ expression (blue). Retinal differentiation (a-Dac, green) starts at the 'firing center' (yellow circle, b) and progresses anteriorly. (d,e) Expression of eyg-lacZ (green) and wingless (a-Wg, red) in ey-GAL4/1XUAS- $N^{E C D}$ (d) and ey-GAL4/2XUAS- $N^{E C D}$ (e) eye discs. (f) Eye disc with $N^{-}\left(N^{\times K 11}\right)$ clones marked by two copies of the GFP (bright green expression) transgene, stained with antibody to $\beta$-galactosidase (eyg-lacZ, red) and Eya (a-Eya, blue). $N^{-}$clones lacked autonomous eyg-lac $Z$ (white arrow) expression and cells with two copies of the $N^{+}$duplication marked by the absence of GFP (filled arrow) had higher levels of eyg-lac $Z$ expression than the heterozygous $\mathrm{N}^{+/-}$tissue (open arrow). Detail showing the single images of GFP (g) and eyg-lacZ (h) expression. (i) Eye disc with $\mathrm{Su}(\mathrm{H})^{\Delta 47} \mathrm{clones}(\mathrm{arrow})$ marked by the absence of GFP (blue) and labeled for Eyg (red). (j-m) Ectopic eyg-lacZ (green) expression is associated with clones expressing the Notch ligands, Delta (UAS-DI; j,k) or Serrate (UAS-Ser; I, m). Clones are marked by the presence of GFP (yellow in $\mathbf{j}$; and purple in I) expression. Marginal clones did not induce eyg, suggesting the presence of a negative regulator of eyg in this region. The high levels of Delta or Serrate can repress eyg-lacZ expression in the clones and the dorsal (D)-ventral $(V)$ region $(\mathbf{k}, \mathbf{m})$. Similar dominant-negative effects of Delta and Serrate proteins occur in the wing and leg discs (reviewed in ref. 3). (n) Eye disc with double $D R^{R e v F 10} S^{R \times 82}$ mutant clones marked by the absence of lacZ (arm-lacZ, red) expression and stained with Eyg (a-Eyg, green) antibody. Note the ectopic Eyg expression (inset) at the edges of the clone. In all figures, posterior is to the right and dorsal is up. 
expressed in D. melanogaster. In contrast, the human PAX6 variant, and its fly counterparts (Ey and Toy), act primarily in specification and differentiation of eye cells in vivo.

\section{RESULTS}

\section{Notch signaling pathway in eye disc growth}

The early development of the fly eye primordium depends on its subdivision into dorsal and ventral compartments (Fig. 1b $)^{3,21,24}$. The localized activation of Notch receptor on both sides of the compartment boundary is believed to establish the dorsal-ventral organizer that controls growth of the entire eye primordium ${ }^{23-25}$. Consistent with this model, several Notch target genes, including four jointed (ref. 25) and Enhancer of split-m $\beta(E(s p l)-m \beta)^{24}$, are expressed prominently on both sides of the dorsal-ventral boundary (see also Fig. 1c). Reducing Notch activity by overexpression of a dominant-negative form of Notch $\left(N^{\mathrm{ECD}}\right)$ leads to a reduction of eye-disc size (Fig. 1d $)^{23-25,34,35}$. In a stronger condition, by overexpression of two copies of $N^{\mathrm{ECD}}$, a more extensive eye-disc loss is observed (Fig. 1e). Analysis of Notch pathway mutants in genetic mosaics supports the idea that the eye-loss defect results from growth defects rather than eye-specification failure (Fig. 1).

Clones of cells carrying a mutated amorphic allele of Notch ( $\left.N^{\mathrm{XK11}}\right)$ produced during the first and early second larval stages expressed the eye-specification marker Eyes absent (Eya; Fig. 1f) and often were smaller than the corresponding $N^{+}$sister clones (or 'twin spots') in the dorsal-ventral region (Fig. 1f,g).

Both the generalized expression of fringe (Fng, encoded by $f n g$ ) and its inactivation arrest eye growth ${ }^{23-25}$ owing to a failure to upregulate the expression of the Notch ligands Delta $(D l)$ and Serrate $(S e r)^{25}$. Accordingly, the consequence of both loss and generalized expression of Fng is reversed through the expression of a ligand-independent activated form of Notch ( $N^{\text {intra. }}$ ref. 24), which results in overgrown eyes ${ }^{23-25,34-36}$.

Generalized expression of Fng under the control of the ey-GAL4 transgene ${ }^{32}$ results in a reproducible 'small eye' defect (Fig. 2a,b), which is equivalent to a hypomorphic $N^{-}$condition (data not shown). In these flies, introduction of a mutated copy of the gene Delta $\left(D l^{\mathrm{X}}\right)$, but not Serrate (Ser ${ }^{\mathrm{RX} 106}$ or Ser ${ }^{\mathrm{RX} 82}$ ), enhanced the defect (Fig. 2c and data not shown). Conversely, introduction of a gain-of-function Notch allele $\left(A x^{\mathrm{M} 1}\right)$ suppressed the phenotype (Fig. 2d). Unlike Notch pathway mutants, the flies with generalized expression of Fng did not have other Notch mutant defects, such as the neurogenic phenotype. Thus, these flies provide an ideal sensitized genetic background in which to investigate specific interactions between the Notch pathway and Pax genes in eye growth.

Figure 2 Eyg lies downstream of and mediates Notch signaling in eye growth. Adult heads of female wild-type flies (a) and combinations between different mutants, UAS transgenes and ey-GAL4 raised at $25^{\circ} \mathrm{C}$ are shown $(\mathbf{b}-\mathrm{I})$.

(b) Generalized Fng expression (UAS-fng) under control of ey-GAL4 results in the formation of very small eyes. (c) Introducing a mutated allele of Delta $\left(D I^{X}\right)$ enhanced the defect resulting in the complete absence of the eyes. (d) By contrast, introducing a gain-of-function Notch allele $\left(A x^{\mathrm{M} 1}\right)$ partially rescues the defect. (e) Coexpressing UAS-eyg and UAS-fng consistently rescued eyes. (f) Overexpression of UAS-eyg also rescued the growth defect caused by $2 x \cup A S-N^{\mathrm{ECD}}$. (g) Overexpression of UAS-toy did not rescue eye growth. Overexpression of UAS-ey occasionally rescued one eye (h), but for the most part, it enhanced the eye-growth defect, resulting in eyes completely missing (i) and concomitant formation of ectopic tiny eyes and palpus-like structures (white arrowheads). (j) Reduction of eyg activity by $50 \%$ resulted in the disappearance of the eye and reduced head in the eyGAL4/UAS-fng flies. (k) Overexpression of Delta (UAS-DI) under control of ey-GAL4 results in overgrown eyes. (I) Introducing two mutated copies of eyg in these flies (UAS-DI, ey-GAL4/+; eyg ${ }^{\left.\text {PXA1 } / \text { eyg }^{X 119}\right)}$ results in the reduction of size or the disappearance of the eye.

\section{Eyg mediates Notch signaling in eye growth}

It has been postulated that Notch signaling is required for the activation of eye-specification regulatory network genes, including ey and to $y^{34,35}$, implying that restoration of expression of these genes might rescue eye growth in the Notch-deficient background. To test this hypothesis, we assayed the eye-growth capability of flies expressing each Pax6 protein, including Eyg and the novel Eyg-like protein (Toe) encoded by the gene twin of eyegone (toe; also known as CG10704), identified by the Berkeley Drosophila Genome Project. Using ey-

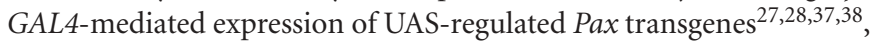
we compared the eye size of flies coexpressing the Pax proteins and Fng with that of flies expressing Fng alone.

Only expression of Eyg fully and consistently rescued the eye-size defect caused by both generalized expression of Fng (Fig. 2e) and of the dominant-negative Notch protein $\left(N^{\mathrm{ECD}}\right.$, Fig. 2f). Toe partially rescued the eye defect (data not shown). By contrast, Toy was unable to bypass the effects of generalized expression of Fng in eye growth (Fig. 2g). Ey partially rescued eye size in some flies (Fig. $2 \mathbf{h}$ ) but more typically enhanced the small-eye defect of Fng and induced the ectopic formation of tiny eyes in different places of the head (Fig. 2i).

We next examined whether reducing the activity of the endogenous Pax genes enhanced the eye defect of reduced Notch activity in the Fng flies. Null ey mutations ${ }^{26,39}$ caused eye loss with inconsistent phenotypes, probably owing to the partially redundant functions of Ey and Toy. The toy allele, toy ${ }^{\text {hdl }}$ (ref. 40) causes a more severe defect but encodes a C-terminally truncated protein that may compete with the other Pax6 proteins in a dominant-negative fashion, precluding any clear inference about Toy function.

Reduction of ey activity by $50 \%$ did not alter eye size in the Fng background (data not shown), indicating that either Ey has no effect on growth or Toy can compensate for the reduction of ey activity. Halving the dose of eyg itself dominantly enhanced the small-eye defect (Fig. 2j), resulting in a considerable size reduction or complete absence of eyes and a smaller head. Overexpression of Delta led to overgrown eyes (Fig. 2k); under this condition, introducing two mutated copies of eyg led to flies without eyes (Fig. 21).
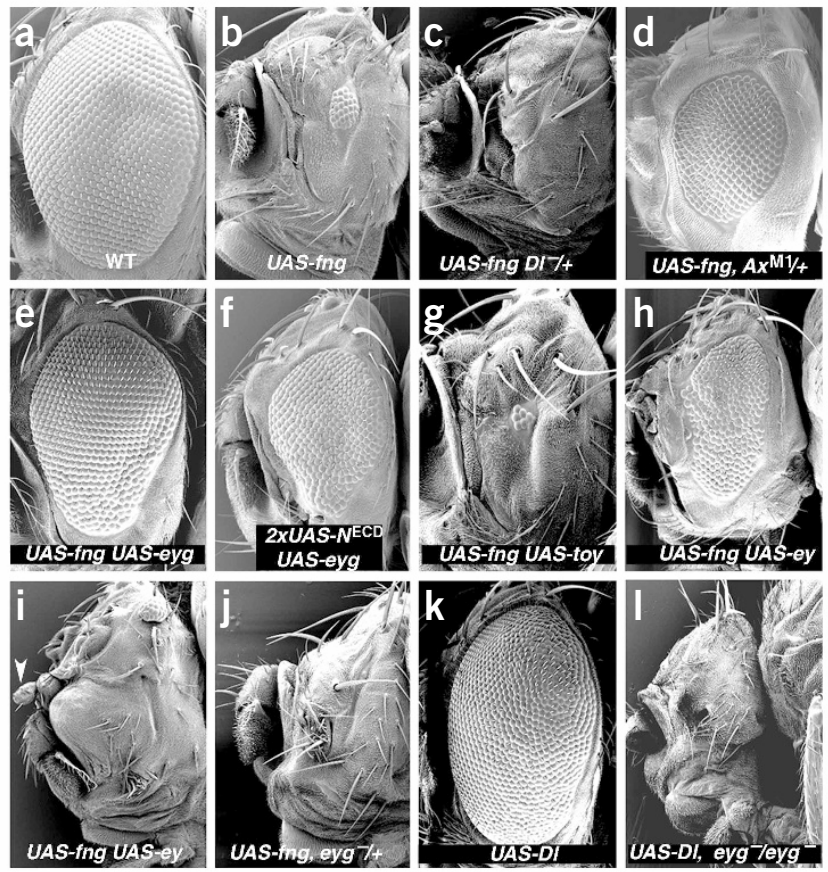


\section{Dose of eyg ${ }^{+}$directly affects eye size}

Our results so far suggest that eyg has the predominant role in regulating eye growth in D. melanogaster. It has been reported that some eygmutant flies retained some eye tissue, as previously observed with null $e y^{-}$(ref. 39) or toy hdl (ref. 40) flies, suggesting that these factors could function redundantly. In flies carrying the eyg alleles that we characterized, however, the eye was completely missing (Fig. 3).

We ranked eyg alleles in a phenotypic series in which the weak combinations led to a variable eye size reduction (Fig. $4 \mathbf{a}, \mathbf{b})$, intermediate combinations resulted in consistent eye loss or tiny eyes (Fig. 4c) and stronger combinations caused head loss and hence were lethal ${ }^{31}$ (Fig. 4d,e). Lethal homozygous combinations were rescued to healthy adulthood with almost normal-size eyes (Fig. $\mathbf{4 f , g , h}$ ) by one copy of an eyg transgene.

Apart from eyg ${ }^{1}$, all new eyg alleles produced various phenotypes unrelated to eye development ${ }^{30,31,37}$ (Fig. 4c,e), which correlated well with the pattern of eyg expression in other imaginal discs (Fig. 3a,d) ${ }^{30,31,37}$.

\section{eyg inactivation prevents eye growth, not specification}

Analysis of eye and antennal discs showed that the loss of eye and head structures in severe $e y g^{-}$alleles could be attributed to defects specific to growth. Flies with all strong and null eyg alleles had normal-size antennal disc parts, but only rudimentary eye disc parts (Fig. 5). eyg ${ }^{\mathrm{P} 20 \mathrm{MD} 1}$ flies had the most severe defects, completely lacking eye discs (Figs. $\mathbf{3 b}$ and $\mathbf{4 d}$ ) and often having only a rudimentary antennal disc (data not shown).

We considered it improbable that the loss of eyg blocked function of eye-specification factors (e.g., if Eyg was upstream of Toy and Ey) for two reasons. First, we found evidence that $e y$ is transcribed in eyg-null mutants and were able to use the eye-specific enhancer of $e y$ ( $e y$ GAL4) to drive expression of the eyg transgene in the eyg- mutant background (Fig. 4g). Second, despite the fact that Ey, and its mouse homolog Pax6, are potent inducers of ectopic eyes in other imaginal discs ${ }^{10,33}$, their overexpression here did not rescue severe eyg alleles (see Methods). This overexpression brought about ectopic small eyes in the remnants of the head and antennal disc anlagen (data not

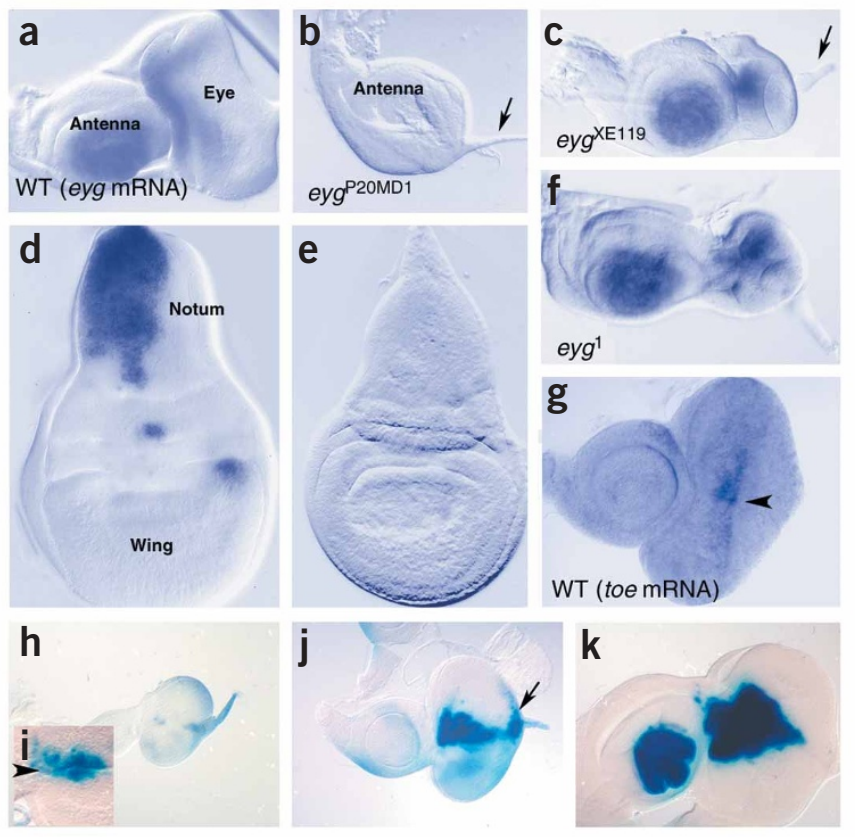

shown; but see Fig. 2i). These findings indicate that eye disc loss associated with eyg is not caused by a failure in specification of the eye.

This conclusion is reinforced by the apparently normal expression in eye discs of flies with severe eyg mutations of other eye-specifying transcription factors, which normally act in concert with Toy and Ey (refs. 10,33), namely eyes absent (eya; Figs. 5f and 6c), sine oculis (so; data not shown) and dachshund (dac; Fig. 5g-i). Misexpression of Eya or $\mathrm{Dac}^{33}$ can trigger ectopic eye formation in other imaginal discs. Consequently, the persistent expression of Ey and Toy and their targets (Eya, So and Dac) in flies carrying severe (Fig. 5) and null (Fig. 6) alleles of eyg indicate that these eye-specification factors cannot compensate for the loss of eyg in eye growth. Thus, the eyg phenotypes (Fig. 5), along with the phenotypes of Eyg overexpression (Fig. 2), suggest that eyg alone (or with some help from the eyg-like gene toe) is the primary contributor to eye growth in the wild type.

\section{eyg is a target of Notch pathway in the eye}

eyg transcripts are expressed, along with ey and toy, in the entire eye and antennal imaginal discs from stage 15 of embryogenesis (refs. 31,33 and data not shown). The expression patterns of eyg and ey mRNAs are independent of each other ${ }^{27,31,33}$, and overexpression of toy did not induce ectopic eyg expression (data not shown). This suggests that expression of eyg and expression of $e y$ and toy are independent of each other, although we cannot exclude the possibility that toy activity in $e y^{-}$mutants suffices to induce eyg.

During the larval stages, ey and toy are expressed uniformly in the eye disc primordium and anterior to the morphogenetic furrow ${ }^{27,33}$. Notably, and in contrast to a recent report ${ }^{31}$, we found that eyg expression was turned off during the early first larval stage, to be reinitiated in a narrow domain along the dorsal-ventral boundary (Fig. $3 \mathbf{h}-\mathbf{k}$ ). Three lines of evidence indicate that this expression domain of eyg, which coincides with the expression of $E(s p l)-m \beta$ (data not shown), requires regulation by the Notch pathway.

First, eyg is not expressed in eye discs with reduced Notch activity $\left(N^{\mathrm{ECD}}\right.$; Fig. 1e $)$ or in clones of cells lacking Notch activity $\left(N^{\mathrm{XK} 11}\right.$; Fig. 1f,h). The protein Suppressor of Hairless $(\mathrm{Su}(\mathrm{H}))$, an integral part of Notch signaling, interacts with the Notch receptor activated by the ligands Delta and Serrate. The complex then translocates into the nucleus, where it induces the expression of target genes, such as $\mathrm{E}(\mathrm{spl})$ complex $(E(s p l)-C)$. Mitotic clones of cells carrying a hypomorphic allele of $S u(H)$ called $S u(H)^{\mathrm{SF} 8}$ or a deletion of the entire gene $E(s p l)-C$ grew normally at any position in the eye disc and expressed eyg (data not shown). By contrast, clones of cells carrying a more severe allele of

Figure 3 eyg $m R N A$ expression patterns in wild-type and mutant third-instar discs. In situ hybridizations with an eyg-specific mRNA probe (a-f) and with a toe-specific mRNA probe (g) are shown. eyg transcript expression in the wild-type eye (a) and wing (d) discs is completely abolished in the corresponding discs $(\mathbf{b}, \mathbf{e})$ of larvae homozygous with respect to the eyg ${ }^{\text {P2OMD1 }}$ allele. eyg expression is reduced but not eliminated in the weak allele eyg $\mathrm{XE}^{119}$ (c) and the original eyg ${ }^{1}$ (f). The arrows point to the optic stalks in $\mathbf{b}$ and c. (g) toe transcripts were expressed also at the dorsal-ventral boundary (arrowhead) of a mid-third larval instar eye disc, but expression was weaker and more confined than that of eyg. (h-k) Evolving expression of eyg as visualized by $\mathrm{X}$-gal staining of $U A S-l a c Z_{\mathrm{nls}} /+$; $P\{$ GawB $\}$ eyg $123 \mathrm{M} 11 /+$ eye discs. Late first or early second larval eye disc showed no X-gal staining (data not shown), suggesting that broad embryonic expression of eyg had been turned off by this stage. Expression of eyg returned during the early/mid second larval stage along the dorsal-ventral boundary $(\mathbf{h}, \mathbf{i})$. The indentation in the disc epithelium marks the dorsal-ventral boundary. The eye disc in $\mathbf{i}$ is slightly older than that in $\mathbf{h}$. Arrow in $\mathbf{j}$ points to eyg expression at the firing center. 
Figure 4 Dose of eyg ${ }^{+}$directly affects eye size. Phenotypic series of eye defects in eyg mutant combinations: weak (a,b), intermediate (c) and severe (d,e). Note the severe grooved thorax phenotype (inset, arrow) of intermediate (c) and severe (e) alleles. (f) Drawing of a D. melanogaster adult head with the structures originating from the eye-antennal disc complex highlighted in gray. (g,h) Rescue by one copy of the transgene eyg in the severe eyg background (UAS-eyg/ey-GAL4; eyg ${ }^{\text {PxA1} / P\{G a w B\} e y g}{ }^{123 M 11}$ ).

$\mathrm{Su}(H)$ rarely contributed to the presumptive anterior dorsal-ventral region (as defined by the expression of eyg). The few clones obtained lacked eyg expression (Fig. 1i). eyg has additional domains of expression in the antennal disc (Fig. 1d,e) that are not related to the eye organizer and are not regulated by Notch.

Second, expression of Notch ligands promotes ectopic eyg expression (Fig. 1 j-m). We generated clones coexpressing the green fluorescent protein (GFP) and ligands using the flip-out technique. Ectopic eyg expression was promoted by $\mathrm{Dl}$-expressing clones only in the ventral region (Fig. 1j,k), where fng is expressed (Fig. 1b). Conversely, eyg expression was induced by the Ser-expressing clones only in the dorsal region (Fig. 11,m), where fng is not expressed.

Third, analysis of mitotic clones carrying severe loss-of-function alleles of the genes Delta and Serrate $\left(D l^{\mathrm{RevF} 10} \mathrm{Ser}^{\mathrm{RX} 82}\right)$ confirmed that both ligands are necessary and sufficient for eyg expression in the eye (data not shown). New borders of eyg expression were sometimes induced at the edges of homozygous $\mathrm{Dl}^{-1-} \mathrm{Ser}^{-1-}$ clones (Fig. 1n). On the basis of these data, we conclude that the regulation of eyg by Notch involves Delta and Serrate and requires the activity of $S u(H)$, but is independent of $E(\mathrm{spl})$-mediated repression of genes.

The regulation and function of Notch in the dorsal-ventral organizers in the fly eye and wing discs is conserved ${ }^{2,3,23-25}$. In the wing, Notch induces the expression of the gene vestigial and the homeobox gene Distal-less in the dorsal-ventral boundary cells ${ }^{3}$. Although vestigial is not conserved, the vertebrate homologs of Distal-less, the Dlx genes, are expressed in the apical ectodermal ridge of the vertebrate limb bud, which acts as a Radical-fringe- and Notch-dependent organizer for limb outgrowth ${ }^{2,3,41}$. Notably, Distal-less and Dlx proteins have a conserved role in appendage morphogenesis (fly limb and antenna; mouse limb and branchial arch $)^{41}$. Thus, the expression of Pax6-like, Eyg, and of Distal-less and Dlx homeodomain proteins in the eye and limb organizers, respectively, provides a plausible mechanism for the specificity of growth response downstream of the generic Notch organizer.

\section{eyg- clones grow poorly in the dorsal-ventral organizer}

A bona fide organizer factor would be required only in the organizing cells for disc growth. In contrast, a factor needed for cell proliferation

Figure 5 eyg inactivation does not impair eye and head specification. Third larval instar eye-antennal discs are labeled for the photoreceptor marker (a-Chaoptin; a-c), a head-promoting gene (a-Wg; d) and the eyespecification markers eyes absent (a-Eya; e,f) and dachshund (a-Dac, $\mathbf{g}-\mathbf{i})$. The ocelli are primitive eyes at the vertex of the head, which still develop in some severe eyg combinations. Dac expression initiates normally $(\mathbf{g})$ but declines in later stages $(\mathbf{h}, \mathbf{i})$. The discs in $\mathbf{h}$ and $\mathbf{i}$ are from the same larvae. Low-frequency pattern duplications (10-12\% in severe combinations) at the extra antenna in (i) occur concurrently with normal eye-specification gene expression in the eye disc. This conclusion contrasts with the proposal that antennal duplications in $U A S-N E C D / e y-G A L 4$ discs result from the loss of eye-anlagen specification ${ }^{36}$. Genotypes are as follows: (a,e) wild-type, (b) eyg $20 \mathrm{MD} 1$, (c,d) eyg $\mathrm{PxA1} / \mathrm{P}\left\{\right.$ GawB eyg $^{\mathrm{M} 26}$, (f) eyg $20 \mathrm{MD} 1 /$ eyg $\mathrm{XE} 119$, (g) eyg ${ }^{\mathrm{PxA} 1}$ and (h,i) $P\{$ GawB $\}$ eyg $158 \mathrm{M} 26$.
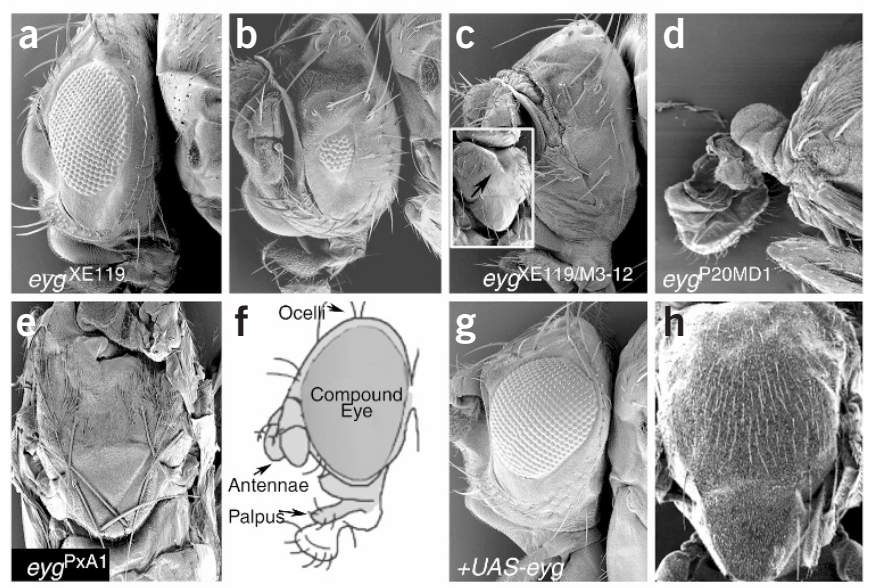

or viability would be required autonomously throughout the entire eye primordia. To distinguish between these two possibilities, we made genetically marked clones of cells lacking eyg activity in different parts of the eye primordium using genetic mosaics. We used eyg $g^{\mathrm{P} 20 \mathrm{MD} 1}$, the most severe allele (hereafter called $e y g^{-}$), for this experiment.

We analyzed 40 mosaic eye discs with 10-20 clones per disc and 120 adult mosaic eyes. We recovered $\mathrm{eyg}^{-1-}$ cells across the entire eyeantennal disc (Fig. 6a), and expression of the markers of neuronal (Elav; Fig. 6a,d) and retinal specification (Eya; Fig. 6c) was normal in $e^{e} g^{-1-}$ cells, suggesting that eyg is dispensable for eye specification, patterning and differentiation. We cannot eliminate the possibilities that some Eyg protein persisted in $e y g^{-}$clones or that Toe compensated for Eyg in these processes.

Mitotic recombination generates genetically marked sister clones ('twin spots') derived from the same mother cell. Clones of eyg cells at the dorsal-ventral boundary were always smaller (Fig. 6a) than their eyg ${ }^{+}$sister clones (Fig. 6a,b). All but 2 of the 128 mosaics that developed normal-size eyes showed the same distribution of clones throughout the eyes (Fig. 6a); the other two had a large central eyg clone with no visible effect (data not shown). The remaining 32 mosaics had overall reduction in eye size due to multiple clones in the dorsal-ventral region ( 8 of 32), nonautonomous outgrowths ( 10 of 32; Fig. 6d,e) or nonautonomous repatterning defects (14 of 32; Fig. 6f). Taken together, these observations suggest that the inactivation of eyg

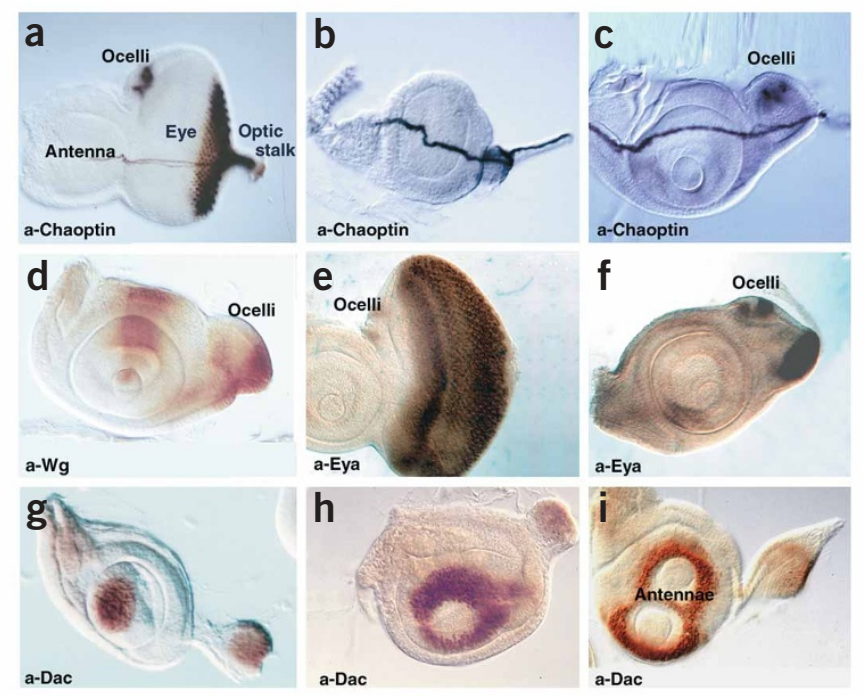


away from the dorsal-ventral organizer has little effect on eye growth, whereas inactivation in the dorsal-ventral region causes growth defects. Thus, eyg function is necessary in regions around the dorsalventral border, where it is induced by Notch signaling.

\section{Boundaries of $\mathrm{eyg}^{+}$and eyg- cells instruct eye growth}

We next examined the effects on growth of larger eyg- clones (Fig. 6f-1). Imaginal discs that were entirely eyg ${ }^{-}$were much smaller and did not differentiate a retina (Fig. 6f), similar to eyg ${ }^{-1-}$ discs (Fig. 5). Notably, mosaic eye discs with $e y g^{+}$cells in the dorsal-ventral region recovered eye-disc growth and differentiation (Fig. 6g,i). The resulting adult mosaic flies had almost normal-size eyes (Fig. $\mathbf{6 j}-\mathbf{l}$ ). These results support a model in which borders between $e y g^{+}$and eyg cells create the growth-promoting boundary. New boundaries that juxtapose $\mathrm{eyg}^{+}$and $e y g^{-}$cells induced a local increase in cell proliferation and pattern duplications in the antennal disc (Fig. 6e,f). Similar outgrowths in the antenna and eye duplications occur after targeted expression of the upstream organizing signal Notch (refs. 34,35) or an eyg transgene (ref. 31 and data not shown).

Eyg is a transcription factor and, therefore, could mediate the activity of the organizer indirectly by driving the expression of diffusible factors (e.g., a morphogen) as postulated for the limbs ${ }^{2-4,42-44}$. We expected that such a diffusible factor produced by the neighboring wild-type cells might rescue growth shortfall in eygmutant clones, but this is not the case (Fig. 6a). We therefore propose an alternative model: the distribution and activation levels of transcription factors (such as Eyg) or their immediate targets (such as short-range inducers $)^{43}$ could be read directly to determine organ growth. In support of this model, we found that the dose of $e y g^{+}$at the dorsal-ventral boundary directly affects eye size (Figs. 3 and 4). Furthermore, boundaries of $e y g^{+}$and $e y g^{-}$cells seemed to instruct tissue growth (Fig. $\mathbf{6 d}, \mathbf{f}-\mathbf{l}$ ), whereas the generalized expression of eyg perturbed eye size, resulting in abnormally small eyes (data not shown). The limbs grew to a significantly larger size than the eye, and perhaps, in this instance, Notch operates by inducing a morphogen to extend the realm of its influence.

\section{Growth and differentiation use distinct PAX6 isoforms}

Ey or Toy and Eyg in the D. melanogaster eye can work independently in cell-fate specification and growth control. This system may be more robust than one in which a single transcription factor is responsible for both growth and specification. This finding raises the possibility that distinct isoforms of mammalian Pax proteins could work similarly to the fly Pax6 and Pax6-like proteins.

The sole PAX6 gene in humans produces two primary isoforms, PAX6(5a) and PAX6, which differ in the inclusion of 14 amino acids encoded by exon $5 \mathrm{a}$ in the N-terminal subdomain of the paired domain ${ }^{10,18-20}$ (Fig. 1a). Like the fly Eyg protein, the human PAX6(5a) isoform binds DNA through the C-terminal subdomain, and both proteins have similar DNA sequence-recognition characteristics ${ }^{18,19,30}$.

We expressed PAX6(5a) or PAX6 under the control of the regionspecific $d p p^{\text {blk }}$-GAL4 driver (Fig. 7). We used the wing pouch to evaluate the effects of the exogenous mammalian PAX6 proteins because the endogenous fly Pax6 proteins are not expressed in this region. Overexpression of human PAX6(5a), like D. melanogaster Eyg (ref. 31)
Figure 6 Boundaries of eyg ${ }^{+}$and eyg cells create new growth-promoting organizers. Mosaic eye discs of the late third larval instar in which the eyg ${ }^{\text {P2OMD1 }}$ mutant tissues are marked by the absence of GFP (a-i) or the absence of red pigments $\left(w^{+} ; \mathbf{j}-\mathbf{I}\right)$. Neuronal differentiation is visualized by Elav (red) in $\mathbf{a}$ and $\mathbf{d}$ and by Eya (green) in $\mathbf{c}$. The yellow arrowheads point to the advancing front of differentiation. $(\mathbf{a}, \mathbf{b})$ Note the disparity in clonal size in eyg ${ }^{\text {P2OMD1 }}$ clones at the dorsal-ventral region (arrowheads) and marginal (arrow) or lateral regions. The bright GFP marks the eyg ${ }^{+/+}$sister clone (asterisks). (b) Eye discs with eyg ${ }^{\mathrm{P} 20 \mathrm{MD} 1}$ clones produced in first instar stage by a single heat-shock pulse generated large eyg+ sister clones (asterisk) and very tiny eyg ${ }^{P 2 O M D 1}$ clones. Mutant clones induced at the same time grew normally in the antennal disc. We noted reduction, but not absence, of cell divisions within homozygous eyg ${ }^{\text {P2OMD1 }}$ mutant clones when compared with eyg $^{+/+}$clones (c). Cell divisions were visualized by pH3 (blue in $\mathbf{c}, \mathbf{f}, \mathbf{g}-\mathbf{i}$ ) expression. (d) Sometimes mutant eyg ${ }^{\text {P2OMD1 }}$ territory (arrow) is associated with de novo nonautonomous growth (arrowhead). (f-I) Eye mosaics induced with the Minute technique ${ }^{49,50}$. A mosaic eye disc consisting almost entirely of homozygous eyg ${ }^{-1-}$ (genotype, eyg ${ }^{\mathrm{P} 20 \mathrm{MD} 1} \mathrm{M}^{+} /$eygP20MD1 $\mathrm{M}^{+}$) tissue marked by the lack of GFP (red) was very small and failed to initiate retinal differentiation. (f) Nonautonomous outgrowth and partial antennal duplication (arrow) as visualized by differential interference contrast (DIC image not shown) and ectopic Wg (green) expression is associated with a small area of heterozygous eyg $^{+/-}$(eyg ${ }^{\text {20MD1 }} \mathrm{M}^{+} /$eyg $^{+} \mathrm{M}^{\text {i55}}$ ) cells. (g-i) Rescued mutant eye discs contain dorsal-ventral areas of heterozygous eyg ${ }^{-1+}$ cells (arrowheads) in an otherwise homozygous eyg ${ }^{-1}$ background and marked by the absence of GFP (red). (h) Single image of the pH3 (blue) expression showed that retinal patterning is restored in the rescued discs. The mosaic disc in $\mathbf{i}$ contains other areas of eyg $^{+-}$cells marked by GFP (red). (j-l) Resulting adult mosaic eyes (compare eye size with that of eyg ${ }^{\mathrm{P} 20 \mathrm{MD1}} /$ eyg $^{+}$heterozygotes in inset). The $\mathrm{w}^{+}$marker in the $u b i$-GFP construct allows the pigmented heterozygous eyg ${ }^{+/-}$tissue to be distinguished from the $w\left(\right.$ GFP-) $^{-}$marker of homozygous eyg $^{-1-}$ tissue. (k) Part of the eye is out of focus (arrow). Invariably, rescued eyes only occurred in mosaics with areas of eyg ${ }^{+/}$cells (pigmented tissue) located within the normal eyg expression domain. Duplications and outgrowths were always connected with borders of eyg ${ }^{+/-}$cells.
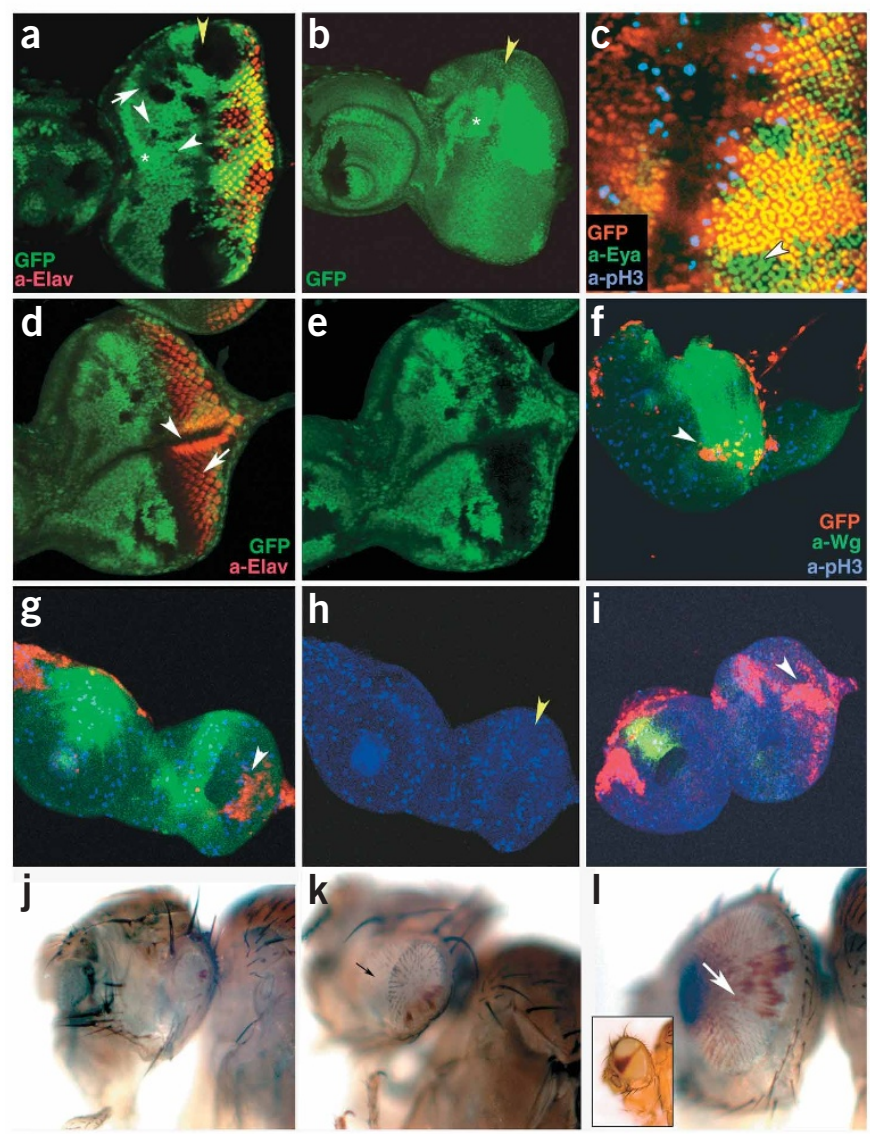
Figure 7 Outgrowths or ectopic retinal differentiation responses to human PAX6(5a) or PAX6 in the developing wing. (a) The control fly expressed UASGFP $_{\text {nls }}$ (green) alone with the transgene $d p p^{b \mid k}$-GAL4, which drives expression in a narrow band of anterior cells along the anterior-posterior (A/P) compartment boundary. Expression of wingless (a-Wg, red) is also shown. (b) The transgenic $d p p^{\text {blk }}$-GAL4 fly coexpressed UAS-lac $Z_{\text {nls }}$ (green) along with the UAS-PAX6(5a) transgene. Induction of UAS-PAX6(5a) expression (green) led to extensive overgrowths (arrows) in both anterior and posterior compartment regions. Mutant cells are marked by the expression of lacZ (green). The disc is also labeled for Wg (red) and the neuronal marker Elav (blue). No ectopic retinal differentiation is observed. (c) Large overgrown wing discs of UAS-PAX6(5a)/dpp blk -GAL4 dissected from larvae $8 \mathrm{~d}$ old and triply labeled with Wg (red), Elav (green) and the posterior cell marker engrailed (en-lac $Z$ in blue). Note the irregular outgrowths, abnormal folds of the disc and the disorganization of anterior and posterior compartments reminiscent of malignant growth. The size of the notum region was not affected by PAX6(5a) protein. (d) Expression of the canonical PAX6 variant primarily induced ectopic retinal differentiation as visualized by clusters of Elav-expressing cells (red). The notum region can show small outgrowths (arrow). All images were taken at the same magnification.

or Toe (data not shown), did not trigger ectopic retinal differentiation in the wing disc (Fig. 7b,c), whereas the PAX6 isoform, like its $D$. melanogaster ortholog Ey/Toy, did so very efficiently under similar conditions (Fig. 7d).

Conversely, overexpression of PAX6 had little or no effect on wing outgrowths (Fig. 7d), suggesting that its effect on growth is secondary to its effect on tissue specification or that PAX6 acts principally through PAX6(5a) protein. In contrast, overexpression of the PAX6(5a) splice isoform induced marked wing outgrowths (Fig. 7b,c). Furthermore, flies expressing PAX6(5a) had an extended larval development (up to five days). Wing primordia dissected from these older larvae typically had huge overgrowths, many abnormal folds, highly irregular organization of anterior and posterior compartments and failure to differentiate (Fig. 7c). These features of discs expressing PAX6(5a) are reminiscent of oncogenic transformation.

\section{DISCUSSION}

Mammals have a single PAX6 gene that produces two primary isoforms, PAX6 and PAX6(5a), by alternative splicing. The genome of D. melanogaster, a less complex organism, has four PAX6-related genes in two groups of paralogs. ey and toy (orthologs of the mammalian PAX6 variant) are located on chromosome IV, whereas eyg and toe (structurally related to the PAX6(5a) variant) ${ }^{10}$, are located on chromosome III. It is not known if some of the D. melanogaster PAX6 genes are redundant, but our analyses of eyg mutants show that Eyg is the primary effector of eye growth. Our results also show that the human PAX6(5a) splice isoform, which lacks the N-terminal subdomain of the paired domain owing to exon insertion, can operate in D. melanogaster by activating the same cellular response (growth) as the D. melanogaster Eyg protein. The human PAX6 isoform, and its fly counterparts Ey and Toy, act principally to specify eye fate. Notably, missense mutations of the N-terminal and C-terminal subregion of the paired domain result in distinct ocular disorders in humans: aniridia, the more severe disorder, is often associated with mutations in the $\mathrm{C}$ terminus ${ }^{10,45}$. These observations are consistent with the view that the distinct roles of PAX6(5a) and PAX6 proteins shown here are not restricted to D. melanogaster. These studies should contribute to our understanding of PAX proteins in dorsal-ventral patterning of the central nervous system, somitogenesis, organogenesis, stem cell biology and human diseases ${ }^{1,5-15,45}$.
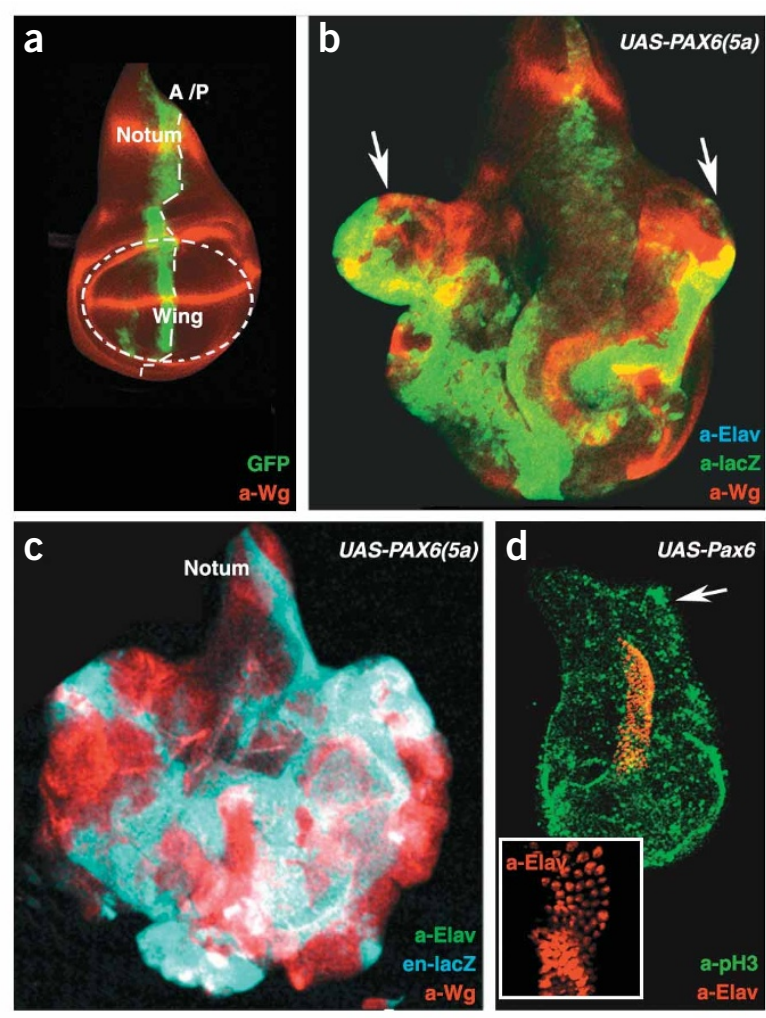

\section{METHODS}

D. melanogaster stocks and transgenes. We used $e y^{\mathrm{JD}}, e y^{11}, e y^{\mathrm{D} 1 \mathrm{Da}}$ and $e y^{\mathrm{EnEH}}$ (ref. 39); eyg ${ }^{\mathrm{S} 2 \mathrm{~A}}$ (ref. 37), eyg $\mathrm{g}^{\mathrm{M} 3-12}$ (ref. 31) and eyg ${ }^{1} ; \mathrm{Df}(3 L)$ iro-2; In (3LR)gv $v^{\mathrm{U}}$; $D f(3 R) E(s p l)^{\text {Delta32.2 }} D l^{\mathrm{X}}$ and $D l^{\mathrm{RevF} 10} ; \mathrm{Ser}^{\mathrm{RX} 82}$ and $\mathrm{Ser}^{\mathrm{RX} 106} ; N^{\mathrm{XK} 11} ; A x^{\mathrm{M} 1}$; $\mathrm{Su}(H)^{\Delta 47}$ and $\mathrm{Su}(H)^{\mathrm{SF} 8}$ (from the Bloomington Drosophila Stock Center). $D f(3 L)$ iro-2 uncovers the Iroquois gene complex, eyg and toe. We used the fol-

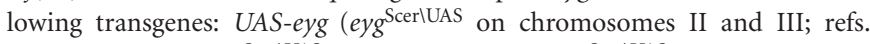

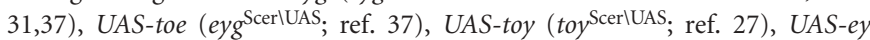
(ey ${ }^{\text {ScerlUAS.UE10 }}$ and $e y^{\text {ScerlUAS.UE11 }}$; ref. 28), UAS-Pax6 (Mmus\Pax6 $6^{\text {ScerlUAS.cHa, }}$ expresses the Sey protein encoded by mouse Pax6; ref. 28), UAS-lac $Z_{\text {nls }}$

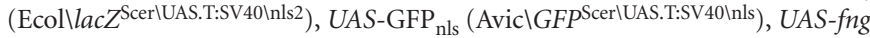
( $f$ g $\left.g^{\text {ScerlUAS.c.Ga }}\right), U A S-D l$ ( $\left.\mathrm{Dl}^{l^{\text {ScerlUAS }}}\right)$, UAS-Ser (Ser ScerlUAS.mg5603 $)$ and UAS$N^{\mathrm{ECD}}$ ( $N^{\mathrm{ECD} . S c e r l U A S}$; two independent insertions on chromosome X and II). In the transgene ey-GAL4 (ScerlGAL4 ${ }^{\mathrm{ey} . \mathrm{PH}}$ ), the Saccharomyces cerevisiae gene $G A L 4$ is expressed under the control of the ey promoter ${ }^{32}$. In the transgene $d p p$ blk-GAL4 (ScerlGAL4 ${ }^{\text {dpp.blk1 }}$ ), GAL4 is expressed under the control of disc

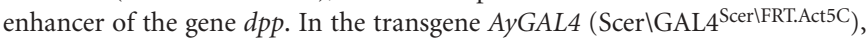
GAL4 is expressed under the control of the Actin-5C promoter. en-lacZ contains the insertion $P\left\{w^{+\mathrm{mc}}=\operatorname{lac}_{\mathrm{C}}\right\} \mathrm{en}^{\mathrm{ryxho} 25}$, which expresses $\beta$-galactosidase in an en pattern, and Iro-lac $Z$ contains the insertion $P\left\{w^{+\mathrm{mc}}=\right.$ lacW $\}$ mirr ${ }^{\text {P69Df7 }}$, which expresses $\beta$-galactosidase in the Iro- $C$ pattern.

Generation of new eyg alleles. eyg ${ }^{\mathrm{XE} 119}$ and $e y g^{\mathrm{XS} 496}$ are both viable X-rayinduced alleles (M.D. and E. Hafen, unpublished results). eyg ${ }^{\mathrm{PxAl}}$ and eyg ${ }^{\mathrm{P} 20 \mathrm{MD} 1}$ are null alleles of eyg and were generated by local hop by $P$-transposon-based mutagenesis using as a starting stock the $P\{$ lacW $\}$ eyg ${ }^{\mathrm{M} 3-12}$ strain (ref. 31), provided by H. Sun (Institute of Molecular Biology, Academia Sinica, Taipei, Taiwan). We cloned DNAs flanking eyg ${ }^{\mathrm{PxA1}}$ and $e y g^{\mathrm{P} 20 \mathrm{MD} 1}$ by inverse PCR. Sequence analysis showed that eyg $g^{\mathrm{PxAl}}$ and eyg ${ }^{\mathrm{P} 20 \mathrm{MD} 1}$ contain $P\{$ lacW $\}$ activity associated with a deletion starting from base -23 relative to the predicted translation start of eyg and extending 11,805 nucleotides downstream of eyg. This deletion was probably present in the original eyg $g^{\mathrm{M} 3-12}$ allele. The deletion does not eliminate the toe transcription unit. eyg ${ }^{\mathrm{PxAl}}$ had two $P\{$ lacW $\}$-element insertions at bases -24 and -147 relative to the translation start of eyg that are in the same transcriptional orientation as eyg. eyg ${ }^{\mathrm{P} 20 \mathrm{MD} 1}$ 
seems to be the result of various imprecise excision or insertion events. An almost complete $P\{l a c W\}$ element in the opposite transcriptional orientation to that of the original $P\{l a c W\}$ in $e y g^{\mathrm{M} 3-12}$ is inserted at base +72 relative to the proximal end of the deficiency. At the right end of this $P$ element, there is a fragment of a $5^{\prime}$ end of probably the original $P$ element in eyg ${ }^{\mathrm{M} 3-12}$. At the left (distal) end, there is a $3^{\prime}$ end of an incomplete $P$ element or a complete $P$ element in the opposite transcriptional orientation. eyg ${ }^{\mathrm{P} 20 \mathrm{MD} 1}$ homozygotes (Fig. 3b) had the most severe defect, completely lacking eye tissue, and we detected no phenotypic differences, at least in the eye, between eyg $g^{\mathrm{P} 20 \mathrm{MD} 1}$ homozygotes and eyg $g^{\mathrm{P} 20 \mathrm{MD} 1} / D f(3 \mathrm{~L})$ iro-2 hemizygotes. We reasoned that the eyg ${ }^{\mathrm{P} 20 \mathrm{MD} 1}$ molecular lesion reduces expression of toe.

$e y g^{123 \mathrm{M} 11}, e y g^{54 \mathrm{~A} 3}, e y g^{\mathrm{M} 26}, e y g^{158 \mathrm{M} 26}$ and $e y g^{76 \mathrm{M} 26}$ are $P\{$ GawB $\}$ eyg alleles. The $P\{G a w B\}$ transgene construct (full genotype, $P\left\{\right.$ Scer $\backslash G A L 4^{w B} w^{+m W . h s}$ EcollampR Ecollori=GawB\}) expresses GAL4 in the eyg pattern. We generated these alleles by targeted transposition, replacing the $P\{l a c W\}$ element in eyg ${ }^{\mathrm{PxAl}}$ mutation with a $P\{G a w B\}$ transgene construct as described ${ }^{46}$. Heterozygous $P\{G a w B\} e y g^{-1+}$ flies dominantly have a weak 'grooved' thorax phenotype and faithfully reproduce eyg expression. Homozygous $P\{$ GawB $\}$ eyg ${ }^{-}$flies have eyedisc defects similar to those of $P\{l a c W\}$ eyg ${ }^{\text {PxAl }}$ flies (data not shown), except that the $P\{$ GawB $\} e y g^{54 \mathrm{~A} 3}$ allele had an earlier lethal phase. Of note, all severe eyg alleles consistently resulted in severe eye-disc reduction.

Generation of the UAS-PAX6 $(5 a)$ transgene. We cloned a 1.5-kb XbaI-XhoI DNA fragment (gift from V. van Heyningen; MRC Human Genetics Unit, Edinburgh, UK) containing full-length human cDNA encoding the PAX6(5a) isoform into a pUAST vector ${ }^{38}$ and transformed it into $w^{1118}$ flies. Expression of the transgene UAS-PAX6(5a) (genotype: $w^{1118}$ PAX6 $(5 a)^{\text {ScerlUAS.t17c })}$ under the control of ey-GAL4 is lethal, and we could not assess whether PAX6(5a) could functionally replace eyg activity in the eye. Therefore, we induced transgene expression using $d p p^{\text {blk }}$-GAL4 or random clones. We generated clones of cells expressing PAX6(5a) using the tub-GAL80 technique ${ }^{47}$ and confirmed that PAX6(5a) could produce outgrowths in any position of the wing (data not shown). We assayed the effects of the canonical PAX6 protein using the Pax6 transgene (ref. 28) because mouse Pax6 is 100\% identical to the human PAX6 variant.

Genetic crosses to rescue eyg using Pax6 transgenes. Unfortunately, eyg activity is required for its own expression in the eye disc, and so we could not use our mutated $P\{G a w B\} e y g^{-}$alleles. Instead we used $e y-G A L 4$ transgene on chromosome II to drive expression of UAS-eyg in the eye tissue combined with the mutated $P\{$ GawB $\}$ eyg alleles to drive expression of UAS-eyg in the remaining eyg patterns. We obtained homozygous eyg flies, rescued by a copy of the transgene UAS-eyg (Fig. 4) by crossing ey-GAL4/CyO; eyg ${ }^{\mathrm{PxAl}} / \mathrm{TM} 3 \mathrm{Sb}$ flies to UAS-eyg/SM5A-TM6B/P\{GawB $\}$ eyg $g^{123 M 11}$ flies. The homozygous eyg flies with a copy of the transgenes UAS-ey or UAS-Pax6 were not rescued. We obtained these flies as follows. First, we crossed $e y-G A L 4 / C y O ; e y g^{\mathrm{PxAl}} / T M 3 S b$ flies to UAS-ey (construct UE11)/SM5A-TM6B/P\{GawB $\}$ eyg $g^{123 M 11}$ flies. Second, we recombined the transgene UAS-Pax6 onto the eyg $\mathrm{g}^{\mathrm{P} 0 \mathrm{MD} 1}$ chromosome and crossed ey-GAL4/SM5A-TM6B/P\{GawB $\} /$ eyg $^{123 M 11}$ flies to UAS-Pax6 eyg ${ }^{\mathrm{P} 20 \mathrm{MD} 1} / \mathrm{TM} 6 \mathrm{~B}$ flies. The mutations Stubble $(\mathrm{Sb})$ and Tubby $(\mathrm{Tb})$ in the TM3Sb and TM6B balancer chromosomes allowed unambiguous identification of the eyg homozygotes by the $\mathrm{Sb}^{+}$or $\mathrm{Tb}^{+}$phenotype of their thoraxes.

Marked clones of mutant cells. We generated clones coexpressing the UAS $G F P_{\text {nls }}$ and UAS-Dl or UAS-Ser using the flip-out technique and generated clones of cells homozygous with respect to loss-of-function mutations using Flipase $(F L P)$-mediated recombination ${ }^{48,49}$, in which we used either transgene hs-FLP (construct, $P\left\{r y^{+t 7.2}=h s-F L P\right\} 112$ ) or transgene ey-FLP (construct, $\left.P\left\{r y^{+\mathrm{t} 7.2}=e y-F L P . N\right\} 2\right)$. We analyzed at least 20 mosaic eye discs of each of the following genotypes: (i) UAS-Dl (or UAS-Ser) flip-out expressing clones: $y$ whFLP112; AyGAL4 UAS-GFPnls/+, P \{lacW $\}$ eyg $g^{\mathrm{PxAl}} /$ UAS-Dl (or UAS-Ser); clones were induced by a single heat shock pulse of $10 \mathrm{~min}$ at $35^{\circ} \mathrm{C} 48-72 \mathrm{~h}$ after egg laying); (ii) Suppressor of Hairless- clones: y w ey-FLP/+; $S u(H) \Delta^{47}$ FRT40A/2xubi-GFP $\{w+m C\}$ FRT40A or ey-FLP/+; Su(H $)^{\mathrm{SF8}}$ FRT40A/2xubiGFP $\{w+m C\}$ FRT40A; (iii) $D l-S e r-$ clones, anti-Eyg: ey-FLP/+; FRT82B $D l^{\mathrm{RevF} 10} \mathrm{Ser}{ }^{\mathrm{RX} 82} / F R T 82 B$ arm-lacZ; (iv) Enhancer of split complex-clones, antiEyg: ey-FLP/+; FRT82B $\mathrm{kar}^{2} \mathrm{ry}^{506} \mathrm{P}\left\{\mathrm{gro} \mathrm{O}^{+}, r y^{+}\right\}$E8 Df(3R) E(spl) $)^{\text {Delta32.2 } / F R T 82 B}$ $u b i-G F P\left\{w^{+m C}\right\}$ (the $D f(3 R) E(s p l)$ deletes the gene groucho ( $g r o$ ) and the $P\left\{g r o^{+}\right.$, $\left.\mathrm{ry}^{+}\right\} E 8$ transgene rescues its activity); (v) $\mathrm{N}$ - clones, eyg-lac $Z$, and anti-Eya: $y$ $N^{\mathrm{XK} 11} h s-F L P 122 / Y ; D p(1 ; 2) w^{\text {ec }} N^{+} \quad F R T 40 A / u b i-G F P\left\{w^{+\mathrm{mC}}\right\} \quad F R T 40 A$; eyg $\mathrm{PxAl}^{\mathrm{Px} /+}$ (FLP-mediated mitotic recombination results in the loss of the $D p(1 ; 2$ $w^{\text {ec }} N^{+}$marked by the presence of two copies of GFP transgene (bright green expression), whereas the absence of GFP expression marks, in this experiment, the wild-type ( $\left.D p(1 ; 2) w^{\text {ec }} N^{+} / D p(1 ; 2) w^{\text {ec }} N^{+}\right)$sister clone); (vi) eyg-clones,

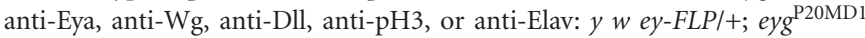
FRT80B/P $\left\{w^{+}\right\} 70 C, F R T 80 B$ (adult mosaic eyes); y $w$ ey-FLP/+; eyg ${ }^{\mathrm{P} 20 \mathrm{MD} 1}$ FRT80B/ubi-GFP $\left\{w^{+\mathrm{mC}}\right\}$ FRT80B (larval eye mosaics); and y w ey-FLP/+; eyg ${ }^{\mathrm{P} 20 \mathrm{MD} 1}$ FRT80B/M $M^{\mathrm{i55}}$ (synonymous $M(3 L) 67 \mathrm{C}$ ), ubi-GFP $\left\{w^{+m C}\right\}$ FRT80B (Minute experiments).

In situ hybridization, antibody staining, microscopy and scanning electron microscopy. We used PCR-amplified fragments as a template for synthesis of $e y g$ - and toe-specific RNA probes. All lethal alleles showed altered expression of eyg transcripts. We treated wild-type and mutant eye-antennal discs together in a single reaction throughout the entire hybridization and staining procedure; we recognized the mutant eye discs by their small size.

We carried out antibody staining as previously described ${ }^{21,24}$. We used the following primary antibodies: mAbdac2-3, mAbEya10H6, mAb4D4, mAb24B10 (Developmental Studies Hybridoma Bank), rabbit pH3, rabbit and mouse $\beta$-galactosidase, antibody to Distal-less, guinea pig antibody to Eyg (gift from N. Azpiazu; Centro de Biología Molecular, Universidad Autónoma de Madrid, Spain). The antibody to Eyg recognizes both Eyg and the Eyg-like protein $\mathrm{Toe}^{37}$. We used secondary antibodies from Jackson and Amersham. We collected images using BioRad MRC 1024 and Leica TCS-NT Confocal microscopes and an Axiophot Zeiss microscope. We acquired scanning electron micrographs of D. melanogaster adults using a Philips SEM (Cambridge, UK) and a XL30 Hitachi (Universidad de Valencia, Spain).

\section{ACKNOWLEDGMENTS}

We thank N. Azpiazu, V. van Heyningen, H. Sun, S. Bray, J. Treisman, A. Baonza, A. Carmena, J. Casal and P. Callaerts for reagents; Bloomington Stock Center for fly stocks; Developmental Studies Hybridoma Bank for antibodies; I. Gutierrez-García, E. Ballesta-Illan and M. Northcote for technical assistance; and P. A. Lawrence, M. Freeman, D. Page, L. A. Garcia-Alonso, M. Millan, F. Casares and F. Viana for critical reading of the manuscript. Part of this work was done in the laboratory of P. A. Lawrence at the Medical Research Council Laboratory of Molecular Biology in Cambridge (UK). This work was supported by grants from Fondo de

Investigaciones Sanitarias and Ministerio de Ciencia y Tecnología from Spain and by a European Molecular Biology Organisation Young Investigator Award to M.D.

\section{COMPETING INTERESTS STATEMENT}

The authors declare that they have no competing financial interests.

Received 14 October; accepted 20 November 2003

Published online at http://www.nature.com/naturegenetics/

1. Martinez, S. The isthmic organizer and brain regionalisation. Int. J. Dev. Biol. 45 , 367-371 (2001)

2. Ohuchi, H. \& Noji, S. Fibroblast-growth-factor induced additional limbs in the study of initiation of limb formation, limb identity, myogenesis, and innervation. Cell. Tissue Res. 199, 247-261 (1999).

3. Irvine, K.D. \& Rauskolb, C. Boundaries in development: formation and function. Annu. Rev. Cell Dev. Biol. 17, 189-214 (2001).

4. Neumann, C. \& Cohen, S. Morphogens and pattern formation. Bioessays 19, 721-729 (1997)

5. Dahl, E., Koseki, H. \& Balling, R. Pax genes and organogenesis. Bioessays 18 , 755-765 (1997)

6. Chi, N. \& Epstein, J. Getting your Pax straight: Pax proteins in development and disease. Trends Genet. 18, 41-47 (2002).

7. Das, P. et al. Haploinsufficiency of PAX9 is associated with autosomal dominant hypodontia. Hum. Genet. 110, 371-376 (2002).

8. Glaser, T. et al. PAX6 gene dosage effect in a family with congenital cataracts, aniridia, anophthalmia and central nervous system defects. Nat. Genet. 7, 463-471 (1994).

9. Ton, C.T. et al. Positional cloning and characterisation of a paired box- and homeobox-containing gene from aniridia region. Cell 68, 491-505 (1991).

10. van Heyningen, V. \& Williamson, K. PAX6 in sensory development. Hum. Mol. Genet. 11, 1161-1167 (2002)

11. Cillo, C., Cantile, M., Faiella, A. \& Boncinelli, E. Homeobox genes in normal and malignant cells. J. Cell Physiol. 188, 161-169 (2001). 
12. Gnarra, J.R. \& Dressler, G.R. Expression of Pax-2 in human renal cell carcinoma and growth inhibition by antisense oligonucleotides. Cancer Res. 55, 4092-4098 (1995)

13. Scholl, F.A. et al. PAX3 is expressed in human melanomas and contributes to tumor cells survival. Cancer Res. 61, 823-826 (2001).

14. Winyard, P.J. et al. The PAX2 transcription factor is expressed in cystic and hyperproliferative dysplastic epithelium in human kidney malformations. J. Clin. Invest. 98, 451-459 (1996).

15. Kroll, T.G. et al. PAX8-PPARgamma1 fusion oncogene in human thyroid carcinoma. Science 289, 1357-1360 (2000).

16. Maulbecker, C.C. \& Gruss, P. The oncogenic potential of Pax genes. EMBO J. 12 2361-2367 (1993).

17. Steinbach, J.P., Kozmik, Z., Pfeffer, P. \& Aguzzi A. Overexpression of Pax5 is not sufficient for neoplastic transformation of mouse neuroectoderm. Int. J. Cancer 15, 459-467 (2001).

18. Czerny, T., Schaffner, G. \& Busslinger, M. DNA sequence recognition by Pax proteins: bipartite structure of the paired domain and its binding site. Genes Dev. 7 2048-2061 (1993).

19. Epstein, J. et al. Two independent and interactive DNA-binding subdomains of the Pax6 paired domain are regulated by alternative splicing. Genes Dev. 8, 2022-2034 (1994).

20. Kozmik, Z., Czerny, T. \& Busslinger, M. Alternatively spliced insertions in the paired domain restrict the DNA sequence specificity of Pax6 and Pax8. EMBO J. 16, 6793-6803 (1997).

21. Cavodeassi, F., Diez del Corral, R., Campuzano, S. \& Dominguez, M. Compartments and organizing boundaries in the Drosophila eye: the role of the homeodomain Iroquois proteins. Development 126, 4933-4942 (1999).

22. Yang, C., Simon, M.A. \& McNeill, H. mirror controls planar polarity and equator formation through repression of fringe expression and through control of cell affinities. Development 126, 5857-5866 (1999).

23. Cho, K.O. \& Choi, K.W. Fringe is essential for mirror symmetry and morphogenesis in the Drosophila eye. Nature 396, 272-276 (1998).

24. Dominguez, M. \& de Celis, J.F. A dorsal/ventral boundary established by Notch controls growth and polarity in the Drosophila eye. Nature 396, 276-278 (1998).

25. Papayannopoulos, V., Tomlinson, A., Panin, V.M., Rauskolb, C. \& Irvine, K.D. Dorsalventral signaling in the Drosophila eye. Science 281, 2031-2034 (1998).

26. Quiring, R., Walldorf, U., Kloter, U. \& Gehring, W.J. Homology of the eyeless gene of Drosophila to the Small eye gene in mice and Aniridia in humans. Science $\mathbf{2 6 5}$ 785-789 (1994)

27. Czerny, T. et al. Twin of eyeless, a second Pax- 6 gene in Drosophila, acts upstream of eyeless in the control of eye development. Mol. Cell 3, 297-307 (1999).

28. Halder, G., Callaerts, P. \& Gehring, W.J. Induction of ectopic eyes by targeted expression of the eyeless gene in Drosophila. Science 267, 1788-1792 (1995).

29. Onuma, Y., Takahashi, S., Asashima, M., Kurata, S. \& Gehring W.J. Conservation of Pax 6 function and upstream activation by Notch signaling in eye development of frogs and flies. Proc. Natl. Acad. Sci. USA 99, 2020-2025(2002).
30. Jun, S., Wallen, R.V., Goriely, A., Kalionis, B. \& Desplan, C. Lune/eye gone, a Pax-like protein, uses a partial paired domain and a homeodomain for DNA recognition. Proc. Natl. Acad. Sci. USA 95, 13720-13725 (1998).

31. Jang, C.C. et al. Two Pax genes, eye gone and eyeless, act cooperatively in promoting Drosophila eye development. Development 130, 2939-2951 (2003).

32. Hazelett, D.J., Bourouis, M., Walldorf, U. \& Treisman, J.E. decapentaplegic and wingless are regulated by eyes absent and eyegone and interact to direct the pattern of retinal differentiation in the eye disc. Development 125, 3741-3751 (1998).

33. Heberlein, U. \& Treisman, J.E. Early retinal development in Drosophila. Fini 2000 , 37-50 (2000).

34. Kurata, S., Go, M.J., Artavanis-Tsakonas, S. \& Gehring, W.J. Notch signaling and the determination of appendage identity. Proc. Natl. Acad. Sci. USA 97, 2117-2122 (2000).

35. Kumar, J.P. \& Moses, K. EGR Receptor and Notch signaling act upstream of Eyeless/Pax6 to control eye specification. Cell 104, 687-697 (2001).

36. Go, M.J., Eastman, D.S. \& Artavanis-Tsakonas, S. Cell proliferation control by Notch signaling in Drosophila development. Development 125, 2031-2040 (1998).

37. Aldaz, S., Morata, G. \& Azpiazu, N. The Pax-homeobox gene eyegone is involved in the subdivision of the thorax of Drosophila. Development 130, 4473-4482 (2003).

38. Brand, A.H. \& Perrimon, N. Targeted gene expression as a means of altering cell fates and generating dominant phenotypes. Development 118, 401-415 (1993).

39. Callaerts, P. et al. Drosophila Pax-6/eyeless is essential for normal adult brain structure and function. J. Neurobiol. 46, 73-88 (2001).

40. Kronhamn, J. et al. Headless flies produced by mutations in the paralogous Pax6 genes eyeless and twin of eyeless. Development 129, 1015-1026 (2002).

41. Panganiban, G. \& Rubenstein, J.L. Developmental functions of the Distal-less/Dlx homeobox genes. Development 129, 4371-4386 (2002).

42. Day, S.J. \& Lawrence, P.A. Measuring dimensions: the regulation of size and shape Development 127, 2977-2987 (2000).

43. Brook, W., Diaz-Benjumea, F. \& Cohen, S. Organizing spatial pattern in limb development. Annu. Rev. Cell Dev. Biol. 12, 161-180 (1996).

44. Serrano, N. \& O'Farrell, P.H. Limb morphogenesis: connections between patterning and growth. Curr. Biol. 7, 186-195 (1997).

45. Azuma, N. et al. Missense mutation in the alternative splice region of the PAX6 gene in eye anomalies. Am. J. Hum. Genet. 65, 656-663 (1999).

46. Sepp, K.J. \& Auld, V.J. Conversion of lacZ enhancer trap lines to GAL4 lines using targeted transposition in Drosophila melanogaster. Genetics 151, 1093-1101 (1999).

47. Lee, T. \& Luo, L. Mosaic analysis with a repressible cell marker for studies of gene function in neuronal morphogenesis. Neuron 22, 451-461 (1999).

48. Xu, T. \& Rubin, G.M. Analysis of genetic mosaics in developing and adult Drosophila tissues. Development 117, 1223-1237 (1993).

49. Newsome, T.P., Asling, B. \& Dickson, B.J. Analysis of Drosophila photoreceptor axon guidance in eye-specific mosaics. Development 127, 851-860 (2000).

50. Morata, G. \& Ripoll, P. Minutes: Mutants of Drosophila autonomously affecting cell division rate. Dev. Biol. 42, 211-221 (1975). 